\title{
Distally Based Sartorius Flap for Soft Tissue Coverage of the Distal Femur
}

\author{
Ciarstan McArdle ${ }^{1}$, Stefan Louette ${ }^{2}$, Daniel Wilks ${ }^{3}$, Peter Giannoudis ${ }^{4}$, Waseem Bhat ${ }^{5}$
}

\author{
Abstract \\ This case report outlines the use of the distal pedicle of the sartorius muscle in the coverage of a large bony and soft tissue defects of the distal \\ femur in a trauma setting. \\ Keywords: Open distal femur, Sartorius flap, Soft tissue defect. \\ Strategies in Trauma and Limb Reconstruction (2021): 10.5005/jp-journals-10080-1521
}

\section{INTRODUCTION}

The incidence of distal femur fractures is relatively rare, accounting for approximately $3-6 \%$ of all adult femoral fracture. ${ }^{1}$ The soft tissue reconstructive options in this region can be challenging. The use of the sartorius muscle as a transposition flap was first described in 1948, to cover exposed femoral vessels post-inguinal lymph node dissection. ${ }^{2}$ It continues to be used for this purpose. Its use for limb salvage and soft tissue coverage of small defects around the knee and upper third of the leg is largely unreported. Here, we present a case using the distally based sartorius flap for soft tissue reconstruction of the distal femur, showing this underreported flap is a viable option in treating skin defects of the thigh and knee.

\section{Case Report}

A 38-year-old gentleman was referred to our unit following debridement, plate fixation and pedicled gastrocnemius flap and split-thickness skin graft of a degloved open femur fracture on the right. He was referred three weeks post-initial injury. The patient initially presented to an out of region major trauma centre with a crush injury to his right thigh from a heavy goods vehicle. He was taken to theatre on the same day for exploration of the degloving injury where the femoral nerve and artery were both intact. The fracture was temporarily fixed with T-Plates. This was followed by a further debridement of the thigh two days later. On day four post-injury, the patient had an open reduction and plate fixation of a distal femur fracture with soft tissue coverage of a medial knee defect using a pedicled gastrocnemius flap and split skin graft.

On arrival, there was a large area of skin necrosis that extended from the proximal thigh and along the anteromedial border and lateral border of the femur, with an underlying deep soft tissue infection (Fig. 1). A healed, grafted gastrocnemius flap covered the medial aspect of the knee. A mature scar was visible on the lateral right thigh from a previous free tissue transfer for chronic osteomyelitis of the ipsilateral tibia five years prior to his current injury.

Radiological imaging showed extensive comminution of the distal femur with malalignment of the posterior aspect of the femur and angulation of the fracture fragment (Fig. 2). Locules of gas were noted in the anterior compartment of the thigh with some subcutaneous oedema.

\footnotetext{
${ }^{1-3,5}$ Department of Plastic and Reconstructive Surgery, Leeds Teaching Hospital Trust, Leeds, West Yorkshire, United Kingdom

${ }^{4}$ Department of Trauma and Orthopaedic Surgery, Leeds Teaching Hospital Trust, Leeds, West Yorkshire, United Kingdom
}

Corresponding Author: Stefan Louette, Department of Plastic and Reconstructive Surgery, Leeds Teaching Hospital Trust, Leeds, West Yorkshire, United Kingdom, Phone: +0113 243 2799, e-mail: stefan.louette@nhs.net

How to cite this article: McArdle C, Louette S, Wilks D, et al. Distally Based Sartorius Flap for Soft Tissue Coverage of the Distal Femur. Strategies Trauma Limb Reconstr 2021;16(2):120-122.

Source of support: Nil

Conflict of interest: None

Revision surgery was undertaken involving removal of all primary metalwork and radical excision of the distal femur and soft tissues, leaving a defect on the anteromedial aspect of the distal femur that extended from the proximal to the distal thigh. Bone fragments, soft tissue and pus specimens were sent for culture and sensitivity. Excision of the full thickness eschar resulted in a soft tissue defect on the anteromedial aspect of the distal femur that extended from the proximal to the distal thigh. Acute shortening and plate fixation of the femur through the pre-existing wound was carried out for fracture stabilisation, with a resultant $8 \times 6 \mathrm{~cm}$ area of bone and exposed metalwork remaining.

Local soft tissue reconstructive options for this patient were limited, as the medial head of gastrocnemius had been harvested for soft tissue coverage of a medial knee defect at the time of the primary surgery. A free tissue ALT flap from the ipsilateral limb had been used to resurface the distal tibia in treatment for a previous osteomyelitis. Alternative free tissue transfer options were considered; however, concern was raised about a suitable donor pedicle with adequate length to allow for primary anastomosis. Intraoperatively it was felt that the distal pedicle of the sartorius muscle was large enough to support a muscle flap, and therefore, the decision was made to utilise the distal portion of the ipsilateral sartorius muscle based within the wound bed to cover the exposed metalwork and bone. Access to the muscle was made through the existing wound that was extended both proximally and distally to allow full access to the muscle at its 


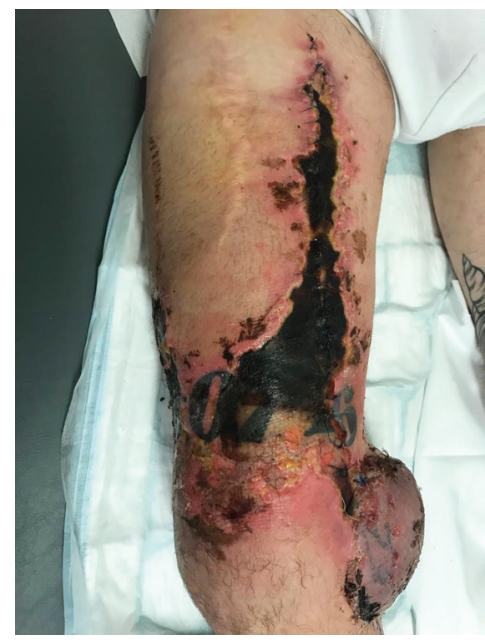

Fig. 1: Right thigh showing a large full thickness eschar extending from the right proximal thigh to the anteromedial aspect of the right distal femur with surrounding cellulitis. A smaller full thickness eschar is seen on the lateral aspect of the distal femur

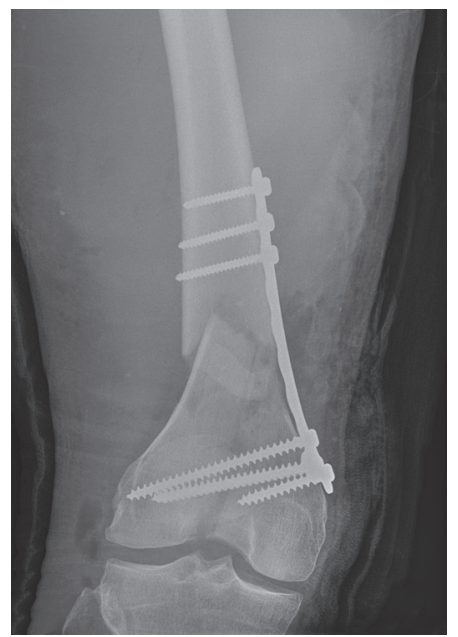

Fig. 2: AP X-ray of the right femur showing extensive comminution of the distal femur with malalignment of the posterior aspect of the femur and angulation of the fracture fragment

proximal and distal insertion. A Doppler probe was used to identify the vascular pedicle. Suitability of the distal pedicle was assessed by vascular clamping, with Ackland clamps, of all other pedicles prior to transposition and inset. The muscle was then transposed and inserted. No evidence of vessel kinking was seen. Clamps were left on for 15 minutes, and the flap assessed by checking bleeding, contractility and colour. The remaining soft tissue was covered with a split-thickness skin graft harvested from the contralateral limb (Fig. 3).

Microbiology specimens showed growth of methicillinresistant staph aureus and vancomycin-resistant enterococcus and acinetobacter radioresistens. On microbiology advice, the patient was treated with a 3-week course of intravenous meropenem and linezolid. This was followed by 3 weeks of oral co-trimoxazole. The patient had an uncomplicated postoperative recovery and wound review on day seven postsurgery showed $100 \%$ graft take (Fig. 4). The patient remained non-weight bearing for 12 weeks, and repeat X-rays at this time showed evidence of callus formation. The patient continues to

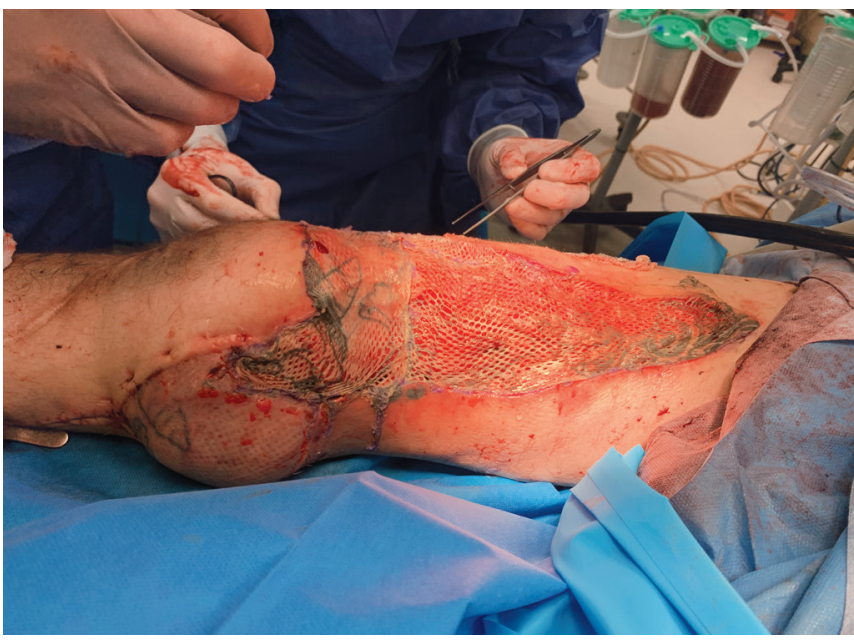

Fig. 3: Soft tissue coverage of the remaining bone and exposed metalwork using the transposed distally based sartorius muscle and split skin graft harvested from the contralateral limb

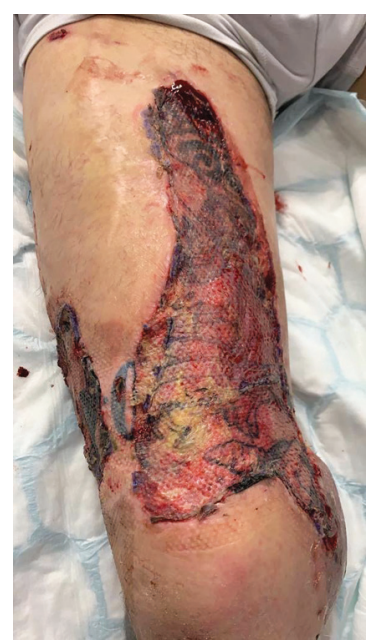

Fig. 4: Wound review two weeks post-surgery. One hundred percent graft take with no evidence of underlying infection

be reviewed in the combined outpatient clinic until the bony union is achieved.

\section{Discussion}

The sartorius muscle is a type IV (Mathes and Nahai classification) long thin biarticular muscle, which originates from the anterior superior iliac spine (ASIS) and runs obliquely to insert onto the medial surface of the proximal tibia. At the hip joint, the muscle acts as a thigh flexor or abductor and external rotator, whilst at the knee, it acts as a knee flexor. ${ }^{3}$

The proximal one-third of the muscle receives its segmental blood supply from the circumflex femoral and superficial femoral artery, whilst the middle one third is supplied by the superficial femoral vessels. The distal third of the muscle is vascularised by the superficial femoral system in addition to the branches of the descending genicular artery. Studies have shown greater than $80 \%$ of muscle survival when isolated on either the proximal or distal pedicle. ${ }^{4}$ 
A cadaveric study published by Manjunath et al focuses specifically on the anatomy of the distal major pedicle of the sartorius flap and the clinical implications for this. They found that in the ten cadavers analysed, the mean location of the distal major pedicle was at $35.25 \mathrm{~cm}$ from ASIS, and the range was between 30.4 and $38.3 \mathrm{~cm}$. They did not find any significant variation between the right and left limbs in individual cadavers. They also found the mean diameter of the distal major pedicle to be $1.54 \mathrm{~mm}^{3}$.

In three clinical cases, they were able to transpose the muscle through $95^{\circ}-125^{\circ}$ of rotation. ${ }^{4}$ These findings were supported in work published by Clavert et al. in which cadaveric unsplit sartorius muscle was transposed through an arc of $100^{\circ}$ of rotation. ${ }^{5}$ An attempted arc of rotation of $155^{\circ}$ to cover a tibial defect led to distal muscle necrosis. ${ }^{3}$

Despite the fact that the sartorius muscle is a long thin muscle, it has the ability to cover soft tissue defects in the distal third of the thigh up to $8 \mathrm{~cm}$ in length without compromising muscle viability, as we found in the case of our patient.

\section{Conclusion}

This case highlights the possibility of treating soft tissue defects of the distal femur and proximal knee defects based on the distal pedicle of the sartorius muscle and that this is a viable option to keep in the reconstructive armamentarium for wounds in this region.

\section{References}

1. Khan AM, Tang QO, Spicer D. The epidemiology of adult distal femoral shaft fractures in a central London major trauma centre over 5 year. Open Orthop J 2017;11:1277-1291. DOI: $10.2174 / 1874325001711011277$.

2. Khalil IM, Sudarsky L. Sartorius muscle 'twist" rotation flap: an answer to flap necrosis. J Vasc Surg 1987;6:93-94. DOI: 10.1067/ mva.1987.avs0060093.

3. Manhunath K, Venkatesh M, Shivaprasad. A distal major pedicle of sartorius muscle flap: anatomical study and its clinical implications. Indian J Plast Surg 2018;51:40-45. DOI: 10.4103/ijps. IJPS_127_17.

4. Habermeyer $P$, Kaiser E, Mandelknow $H$, et al. Anatomy and clinical aspects of sartoriusplasty. Handchir Mickrochir Plast Chir 1987;19:21-24.

5. Clavert P, Cognet J, Baley S, et al. Anatomical basis for distal sartorius muscle flap for reconstructive surgery below the knee. J Plast Reconst Aesth Surg 2008;61:50-54. DOI: 10.1016/j. bjps.2006.01.059. 